Lepr Rev (2000) 71, 363-368

\title{
Predisposing factors for recurrent skin ulcers in leprosy
}

\author{
HEINKE KUNST \\ Tropeninstitut, Gottingen, Germany, and Marie Adelaide Leprosy \\ Centre, Karachi, Pakistan
}

Accepted for publication 16 June 2000

\begin{abstract}
Summary This study was designed to determine the factors associated with recurrence of leprosy ulcers. Between April and August 1992, 55 consecutive leprosy patients admitted with skin ulcers were studied. Factors predisposing to recurrence, e.g. patient's age, disease duration, ulcer site, ulcer depth and physical deformity (taking into account neuromuscular and skeletal damage) were evaluated. Ulcer recurrence occurred in 40/55 (75\%) patients. Recurrent ulceration was associated with location in the lower extremity $(P=0.02)$, where recurrences were more common in the midfoot and heel $(P=0 \cdot 01)$. Recurrence was also associated with severity of physical deformity $(P=0.01)$, which increased the odds of recurrent ulceration by 4.2 times (95\% confidence interval, 1.01-18.3). The severity of physical deformity itself was associated with the age of the patient $(P=0.04)$ and the disease duration $(P=0.02)$. In conclusion, there is a need to focus on identification of risk factors for recurrent leprosy ulceration. Targeted prevention strategies would be required if morbidity associated with recurrent skin ulceration is to be avoided.
\end{abstract}

\section{Introduction}

With the introduction of new therapeutic regimens, leprosy can now be cured. However, complications of the disease such as sensory loss, muscle palsy, absorption of extremities and recurrent ulcers still lead to substantial morbidity. ${ }^{1}$ Of the $10-12$ million leprosy patients in the world, it is estimated that 1.8 million suffer from skin ulcers, ${ }^{2}$ and ulcers remain the most common reason for hospitalization in these patients. ${ }^{3}$ Recurrent ulceration, in particular, also carries a risk of development of squamous cell carcinoma. ${ }^{4}$ Factors predisposing to recurrence, therefore, need to be elucidated with view to prevention. Literature on predisposing factors for ulceration in leprosy is limited. ${ }^{5,6}$ In particular, little attention has been given to deformities and their effect on recurrence of ulceration. The objective of this paper was to study the predisposing factors associated with recurrence of leprosy ulcers and to determine the effect of site and severity of physical deformity on ulceration. 


\section{Materials and methods}

During the period April to August 1992, 55 consecutive leprosy patients with skin ulcers requiring hospitalization were evaluated at the Marie Adelaide Leprosy Centre, Karachi, Pakistan. During the course of their illness, all patients had received regular health education by trained teachers and each patient had been given advice about adequate footwear and had been offered special orthopaedic shoes. The suggested methods of prevention included daily inspection of anaesthetic hands and feet for skin injuries, regular baths and care with Vaseline afterwards to restore loss of seborrhoeic function.

On recruitment in this study, a detailed history including disease activity and duration; ulcer localization and recurrence; and previous treatments and complications were noted for each patient. Information on the aetiology of the ulcers was generally not available due to the long time lag between ulceration and hospitalization. Examination was conducted to assess the neurological system, musculoskeletal system and the form and depth of each ulcer. Loss of pain sensation was examined by using a sterile pin. Patients were asked to close their eyes and their response to sharp stimuli was noted. Touch and light pressure sensation were also examined by using a wisp of cotton wool. The motor system was examined for musculoskeletal function and deformity. Muscular functions were assessed including tone, power and reflexes. Severity of physical deformity was graded according to a uniform classification, ${ }^{7}$ taking into account neuromuscular and skeletal damage as shown in Table 1.

The factors associated with recurrence of ulceration were elucidated using patient as the unit of analysis. This formed the basis for testing our primary research hypothesis regarding the effect of physical deformity on recurrence of ulceration. Secondary analyses were conducted using ulcer as the unit of analysis. Continuous variables were assessed for differences with Student's $t$-test for two groups and analysis of variance was used for multiple comparisons. Proportions were assessed for differences using the Chi-square test (with Yates' correction when expected cell value was $<5$ ) and their trends were analysed with Chi-square for trend. In addition, the association of deformity with site of ulceration was explored in detail for ulcers on the planter aspect of the foot. A two-tail $P$-value of $<0.05$ was regarded as significant. Epi-info Software ${ }^{8}$ was used for statistical analysis.

Table 1. Classification of physical deformity in leprosy

\begin{tabular}{|c|c|c|}
\hline Grade* & Hand deformity & Foot of deformity \\
\hline Grade 1 & Loss of sensitivity & Loss of sensitivity \\
\hline Grade 2 & Ulcers and wounds & Ulcers and wounds \\
\hline Grade 3 & $\begin{array}{l}\text { Mobile minimal clawhand, slight bone } \\
\text { absorption }\end{array}$ & $\begin{array}{l}\text { Hyperextension of first toe, mobile claw toes, } \\
\text { slight bone absorption }\end{array}$ \\
\hline Grade 4 & $\begin{array}{l}\text { Mobile complete clawhand, moderate bone } \\
\text { absorption }\end{array}$ & $\begin{array}{l}\text { Fixed claw toes, flat foot, moderate bone } \\
\text { absorption }\end{array}$ \\
\hline Grade 5 & $\begin{array}{l}\text { Fixed complete clawhand, wrist drop, severe } \\
\text { bone absorption }\end{array}$ & $\begin{array}{l}\text { Contracture of ankle joint, foot drop, severe } \\
\text { bone absorption }\end{array}$ \\
\hline
\end{tabular}

* Based on Kunst (1993). 


\section{Results}

Out of a total of 55 leprosy patients with skin ulcers, there were 40 male and 15 female patients. Their mean age was 49 years (SD 17.4) and the mean duration of disease was 14 years (SD 10; range 1-35). Recurrent ulcers occurred in 40/55 (73\%) patients. Mean time to recurrence was 7 years (SD 5.8; range 1-24). As shown in Table 2, patients with recurrences were older (mean age 50.2 years versus 45.0 years) and had longer duration of illness compared to those without recurrence ( $16 \cdot 1$ years versus $10 \cdot 9$ years); these differences were not statistically significant. However, there was a significant trend towards higher odds of recurrence with worsening grade of physical deformity $(P=0 \cdot 01)$. Severity of deformity increased with age $(P=0.04)$ and disease duration $(P=0.02)$. Compared to patients with deformity grade $1-2$, those with grade $3-5$ had higher odds of having recurrent ulcers (odds ratio $4 \cdot 2,95 \%$ confidence interval $1 \cdot 01-18 \cdot 3, P=0 \cdot 02)$.

The total number of ulcers was 82 , nine $(11 \%)$ in upper extremities and $73(89 \%)$ in lower extremities. Of the 82 ulcers, 54 (66\%) were recurrent. As shown in Table 3, recurrences were more common in the lower extremity compared to upper extremity (51/73 versus $3 / 9$, $P=0.02)$. In the upper extremity $6 / 9(67 \%)$ ulcers occurred on the hand. In the lower extremity $57 / 73(78 \%)$ were localized to the plantar aspect of the foot. Of these, 40/57 (70\%) occurred on the forefoot, $7 / 57(12 \%)$ on the midfoot, and 10/57 (17\%) on the heel. In the foot, recurrence was more often in the midfoot and heel compared to forefoot $(16 / 17$ versus $23 / 40, P=0 \cdot 01)$. The ulcers were superficial in 51/82 (62\%), deep in 22/82 (27\%) and had sinus formation in $9 / 82(11 \%)$ cases. In the lower extremity, deep ulcers accounted for $7 / 40(17 \%)$ forefoot ulcers, $5 / 7(70 \%)$ midfoot ulcers and 8/10 (80\%) heel ulcers. Sinus formation occurred in 6/40 (16\%) forefoot ulcers and 3/6 (50\%) hand ulcers. Depth of ulceration was not associated with recurrence $(P=0 \cdot 25)$. The relation of grade of physical deformity with recurrence of ulceration, using ulcer as the unit of analysis, showed a statistically non-significant trend toward recurrence $(P=0 \cdot 1)$. Compared to ulcers associated with deformity grade $1-2$, those with grade $3-5$ had higher odds of having recurrent ulcers (odds ratio $2 \cdot 2,95 \%$ confidence interval $0 \cdot 8-6 \cdot 2, P=0 \cdot 1$ ).

Table 2. Characteristics of leprosy patients with skin ulcers according to recurrence

\begin{tabular}{lccc}
\hline & $\begin{array}{c}\text { Recurrent ulcer } \\
(n=40)\end{array}$ & $\begin{array}{c}\text { Non-recurrent ulcers } \\
(n=15)\end{array}$ & $P$-value \\
Characteristics & $50 \cdot 2$ (SD 17.5) & $45 \cdot 0$ (SD 18.5) & NS \\
\hline Patient's age (years) & $16 \cdot 1$ (SD 10.4) & $10 \cdot 9$ (SD 9.7) & $0 \cdot 09$ \\
Duration of illness (years) & & & \\
Patient's sex & 30 & 10 & NS \\
Male & 10 & 5 & \\
Female & & 7 & $0 \cdot 01$ \\
Distribution of patients according to grade of physical deformity* & 3 & \\
Grade 1-2 & 7 & 2 & \\
Grade 3 & 4 & 3 & \\
Grade 4 & 7 & 22 & \\
Grade 5 & & & \\
&
\end{tabular}

* See Table 1 for detailed description for physical deformity grading. NS $=$ not significant. 
Table 3. Site and physical deformity associated with leprosy ulcers according to recurrence

\begin{tabular}{lccc}
\hline & $\begin{array}{c}\text { Recurrent ulcer } \\
(n=54)\end{array}$ & $\begin{array}{c}\text { Non-recurrent ulcers } \\
(n=28)\end{array}$ & $P$-value \\
Characteristics & 3 & 6 & \\
\hline Site of recurrence & 51 & 22 & 0.02 \\
Upper extremity & & \\
Lower extremity & 23 & 17 & 0.01 \\
Localization of 57 ulcers in planter aspect of lower extremity & \\
Forefoot & 16 & 1 & \\
Heel and midfoot & 12 & 7 & \\
Distribution of ulcers according to grade of physical deformity* & \\
Grade 1 & 5 & 7 & \\
Grade 2 & 7 & 5 & \\
Grade 3 & 22 & 6 & \\
Grade 4 & 8 & 5 \\
Grade 5 & & \\
& & & \\
\hline
\end{tabular}

* See Table 1 for detailed description for physical deformity grading.

The relation of specific deformities to site of ulceration was explored. In the plantar aspect of the lower extremity, this analysis revealed that flat foot was more often associated with midfoot and heel ulceration than with metatarsal head ulcers $(P<0 \cdot 0001)$, whereas claw foot, foot drop and absorption were not associated with the site of ulcer $(P<0 \cdot 05)$ (Table 4$)$.

\section{Discussion}

In this study, ulcer recurrence was found to be associated with location in the lower extremity, where recurrences were more common in the midfoot and heel; and with severity of physical deformity, which substantially increased the odds of recurrent ulceration in patients with

Table 4. Relation between physical deformity of the lower extremity and foot ulcers location on the planter aspect $(n=57)$

\begin{tabular}{lcccc}
\hline & \multicolumn{4}{c}{ Associated deformity $(n)$} \\
\cline { 2 - 5 } Site of ulcer & Bone absorption $^{1}$ & Claw foot $^{1}$ & Flat foot $^{2}$ & Foot drop \\
\hline Metatarsal heads* $(n=25)$ & 13 & 14 & 6 & - \\
Toes $(n=15)$ & 1 & 7 & - & 1 \\
Heel $(n=10)$ & 4 & 5 & 5 & - \\
Mid-foot $(n=7)$ & 6 & 5 & 6 & - \\
\hline
\end{tabular}

Data presented as number of ulcers; sum of column data exceeds totals because more than one deformity was associated with several ulcers.

* At first metatarsal head there was hyperextension of first toe in $9 / 16(56.3 \%)$ ulcers.

${ }^{1}$ Claw foot and absorption not associated with site of ulceration $(P<0.05)$.

${ }^{2}$ Flat foot associated more frequently with midfoot or heel ulcers than with metatarsal head ulcers $(11 / 17$ versus $6 / 25, P=0 \cdot 009)$. 
more severe grade of deformity. The severity of physical deformity was associated with the patient's age and disease duration.

In determining the conformity of the general findings of this study with other reported literature, we found consistency with regard to the observation of male predominance ${ }^{9}$ and advanced patient age. ${ }^{10}$ Moreover, the distribution of ulcers in the plantar aspect of the foot in this study is concordant with findings of other series. ${ }^{11} \mathrm{We}$ also found an association between midfoot ulcers and a loss of foot arch, which has been described as a predisposition. ${ }^{11}$ However, the lack of an association between site of ulcer and claw foot, foot drop and absorption is perhaps due to type II statistical error (inability to find an association when one actually exists) related with the small sample size of our study. The relation of age with severity of physical deformity found in our study was also consistent with prevailing evidence. $^{10}$

The main finding of our study was the relation of physical deformity with recurrence of ulceration. In studying this association, we could also have used the WHO classification, which is an indicator for an overview of the frequency of deformities in a leprosy programme. However, the WHO classification is not considered to be a good indicator for the assessment of individual patients. ${ }^{12}$ Therefore, we used a modified classification. ${ }^{7}$ This revealed a strong association with recurrent ulceration using patient as the unit of analysis (odds ratio for recurrence in relation to severity of deformity $4.2,95 \%$ confidence interval $1 \cdot 01-18 \cdot 3, P=0 \cdot 02$ ). This association was strengthened by the strong trend in the secondary analysis using ulcer as the unit of analysis (odds ratio for recurrence in relation to severity of deformity $2 \cdot 2,95 \%$ confidence interval $0 \cdot 8-6 \cdot 2, P=0 \cdot 1$ ). As our primary hypothesis was based on patient as the analysis unit, we are confident that our results are not spurious.

The relationship of occupation and health education with leprosy ulcers has been analysed in a separate study. ${ }^{13}$ This analysis has shown that high grade physical deformity was associated with unemployment and there was a trend of higher unemployment among those with recurrent ulcers. With regard to health promotion, there was poor compliance with advice about protective footwear and care of insensitive extremities. Level of knowledge about the leprosy ulcers and use of prevention strategies was also inadequate despite health promotion. For example, with regard to use of footwear, it was evident that patients only realized the necessity of protective shoes after a deformity had developed.

It has been shown that surgical treatment of deformities can contribute to elimination of predisposing factors for recurrent ulceration. ${ }^{4}$ This is of importance, in particular, because duration of recurrent ulceration has a significant influence on malignant degeneration as epidemiological studies have shown that up to $2 \%$ of patients with a mean ulceration time of 12 years develop squamous cell carcinoma. ${ }^{14}$ Since many patients with chronic ulceration are not supervised regularly, malignant change can be easily overseen. ${ }^{15}$ There are limited resources to allow full development of people affected with leprosy, ${ }^{16}$ so if morbidity associated with recurrent skin ulceration is to be avoided, then targeted prevention strategies would be required. Our study indicates that there is a need to focus on identification and correction of physical deformities that predispose to recurrent ulceration.

\section{Acknowledgements}

The author is grateful to Professor W. Bommer of the Tropeninstitut, Gottingen, Germany for his support and supervision in her doctoral thesis work on which this paper is based; to 
Drs T. Chiang and R. Pfau of the Marie Adelaide Leprosy Centre, Karachi, Pakistan for allowing her to study their patients; and to the patients themselves who willingly consented to participate in this work.

\section{References}

1 Noordeen SK, Paaniker VK. Leprosy. In: Cook GC (ed) Manson's tropical diseases. WB Saunders, London, 1996, pp 1016-1044.

2 Hammond CJ, Klenerman P. Protective sensation in the foot in leprosy. Lepr Rev, 1988; 59: 347-354.

3 Yasawant K, Malhotra MD. Role of topical phenytoin trophic ulcers of leprosy in India. Int J Lepr, 1991; 59: 337-338.

4 Bourrel P. Surgical rehabilitation. Lepr Rev, 1991; 62: 241-255.

${ }_{6}^{5}$ Fritschi EP. Surgical reconstruction and rehabilitation in leprosy. Wright, Bristol, 1984.

6 Pring DJ, Casabianca N. 'Dorsal incision' the treatment of complicated forefoot ulcers in the anaesthetic foot. Lepr Ind, 1983; 55: 49-59.

7 Kunst H. Skin ulcers in leprosy patients in Karachi, Pakistan: predisposing factors for recurrence of ulcers (MD thesis). University of Göttingen, Göttingen, Germany, 1993.

${ }^{8}$ Dean AG, Dean JA, Coulombier D et al. Epi Info, version 6: a word-processing, database, and statistics program. Centers for Disease Control and Prevention (CDC), Atlanta, Georgia, 1994.

9 Max E, Shepard DS. Productivity loss due to deformity from leprosy in India. Int J Lepr, 1989; 57: 476-482.

${ }^{10}$ Mishra B,Ramu G. Leprosy deformities in an epidemiological study in a rural area of Rajasthan. Ind J Lepr, 1988; 60: $53-62$.

11 Price JE. A study of leprosy patients with deformities, and implications for the treatment of all leprosy patients. Lepr Rev, 1983; 54: 129-137.

12 Watson JM. Disability control in a leprosy programme. Lepr Rev, 1989; 60: 169-177.

${ }^{13}$ Kunst H. Relationship of skin ulcers and physical deformity with employment status and compliance with health promotion in leprosy. JPMA 2000 (in press).

14 Richardus JH, Smith TC. Squamous cell carcinoma in chronic ulcers in leprosy: A review of 38 cases. Lepr Rev, 1991; 62: 381-388.

15 Ozkan T, Yuksel A, Saylan T et al. Carcinoma in plantar ulcers of leprosy patients: a report of 4 cases from Turkey. Lepr Rev, 1988; 59: 356-357.

16 Walter CS. Social aspects and rehabilitation. Lepr Rev, 1999; 70: 85-94. 